\title{
Convergence of Difference Methods for Initial and Boundary Value Problems with Discontinuous Data
}

\author{
By Bruce Chartres* and Robert Stepleman**
}

\begin{abstract}
This paper extends the classical convergence theory for numerical solutions to initial and boundary value problems with continuous data (the right-hand side) to problems with Riemann integrable data. Order of convergence results are also obtained.
\end{abstract}

1. Introduction. The purpose of this paper is to show that difference methods for solving initial and boundary value problems will converge in a variety of cases where the data (the right-hand side) is not well behaved in the classical sense.

To illustrate this basic idea, we consider two problems:

1. First, we look at Euler's method for solving initial-value problems on [0, 1]:

$$
y^{\prime}(t)=f(t, y(t)), \quad y(0)=y_{0} .
$$

Classically, for convergence, it is assumed that $f$ satisfies a Lipschitz condition in the second variable and $y(t)$ is continuously differentiable (see e.g., Henrici [4], [5]) or, at least, piecewise continuously differentiable (see Goodman [3] or Zverkina [7]). We obtain the following results: If $f$ is a bounded Riemann integrable function along the trajectory and satisfies a Lipschitz condition in its second argument, then Euler's method converges; and, if $f$ is of bounded variation along the solution trajectory, then the convergence is of order $h$.

2. Second, we look at the standard simple difference method for solving the boundary value problem on $[0,1]$ :

$$
y^{\prime \prime}(t)=f(t, y(t)), \quad y(0)=a, \quad y(1)=b .
$$

In general, for convergence, it is assumed that $y(t) \in C^{2}[0,1]$ (see e.g. Lees [6]). We obtain convergence results under assumptions on $f$ of the same type as in (1).

2. Convergence Results 1 . Let $N$ be some positive integer and $h=1 / N$. Set $t_{n}=n h, n=0,1, \cdots, N$; then Euler's method for solving $(1.1)$ on $[0,1]$ is given by

$$
y_{n+1}=y_{n}+h f\left(t_{n}, y_{n}\right), \quad n=0, \cdots, N-1 .
$$

The following theorem gives the convergence properties of (2.1) to solutions of (1.1) as $h \rightarrow 0$, where, by a solution to (1.1), we mean an absolutely continuous $y(t)$ on $[0,1]$ which satisfies the initial condition, and the derivative $y^{\prime}(t)$ equals $f$ every-

Received July 13, 1970, revised April 5, 1971.

AMS 1969 subject classifications. Primary 6561, 6562.

Key words and phrases. Convergence, Euler's method, difference method, discontinuous data, boundary value.

* This work was supported by Grant GJ-28 from the National Science Foundation.

** This work was supported by Grant NONR-474(14) from the Office of Naval Research.

Copyright (c) 1971, American Mathematical Society 
where except on a set of Lebesgue measure zero (see e.g. Coddington and Levinson [1, p. 42]).

THEOREM 2.1. Suppose the solution of (1.1) exists, where $f$ is a bounded Riemann integrable function along the solution trajectory, and there exists $k<\infty$, such that ${ }^{* * *}$ for all $t \in[0,1], a$ and $b$ real

$$
|f(t, a)-f(t, b)| \leqq k|a-b| .
$$

Then

$$
\left|y_{n}-y\left(t_{n}\right)\right| \rightarrow 0 \text { uniformly, as } h \rightarrow 0, h n \rightarrow t \in[0,1] \text {, }
$$

where $y(t)$ is the exact solution of (1.1). If, in addition, $f$ is of bounded variation along the solution trajectory, then

$$
\left|y_{n}-y\left(t_{n}\right)\right|=O(h) .
$$

Proof. Define the local truncation error $\tau_{n}$ by

$$
h \tau_{n+1}=y\left(t_{n+1}\right)-y\left(t_{n}\right)-h f\left(t_{n}, y\left(t_{n}\right)\right), \quad n=0, \cdots, N-1,
$$

where $y(t)$ is the exact solution of (1.1). Observe

$$
\left|y_{1}-y\left(t_{1}\right)\right|=h\left|\tau_{1}\right|
$$

and by (2.2),

$$
\left|y_{n}-y\left(t_{n}\right)\right| \leqq(1+h k)\left|y_{n-1}-y\left(t_{n-1}\right)\right|+h\left|\tau_{n}\right|, \quad n=2, \cdots, N .
$$

Hence,

$$
\begin{aligned}
\left|y_{n}-y\left(t_{n}\right)\right| & \leqq h \sum_{j=0}^{n-1}(1+h k)^{i}\left|\tau_{n-i}\right| \leqq e^{k} \sum_{i=0}^{n-1} h\left|\tau_{n-i}\right| \\
& \leqq e^{k} \sum_{i=0}^{n-1}\left|\int_{t_{j}}^{t_{j+1}} f(t, y(t)) d t-h f\left(t_{i}, y\left(t_{i}\right)\right)\right| \\
& \leqq e^{k} \sum_{j=0}^{n-1} h\left|f_{i}^{*}-f\left(t_{i}, y\left(t_{i}\right)\right)\right|, \quad n=1, \cdots, N,
\end{aligned}
$$

where

$$
m_{i} \equiv \inf _{t \in\left[t_{i}, t_{i+1}\right]} f(t, y(t)) \leqq f_{i}^{*} \leqq \sup _{t \in\left[t_{j}, t_{j+1}\right]} f(t, y(t)) \equiv M_{i} .
$$

Thus,

$$
\left|y_{n}-y\left(t_{n}\right)\right| \leqq e^{k} h \sum_{i=0}^{n-1}\left(M_{i}-m_{i}\right) \leqq e^{k}\left(S_{1}-S_{2}\right),
$$

where $S_{1}$ and $S_{2}$ are, respectively, the upper and lower Riemann sums for $\int_{0}^{1} f(t, y(t)) d t$ over the partition $\left\{t_{0}, \cdots, t_{N}\right\}$. Since $f$ is Riemann integrable on the solution curve, (2.3) follows. If, in addition, $f$ is of bounded variation on the solution curve, then

*** G. Dahlquist has pointed out that the results of this theorem hold when this condition is replaced by the weaker one-sided Lipschitz condition $|a-b+h(f(t, a)-f(t, b))| \leqq(1+k)|a-b|$; (concerning one-sided Lipschitz conditions, see Dahlquist [2]). 
(2.4) follows immediately as a consequence of the uniform boundedness of the sum in (2.7) over all partitions of $[0,1]$.

3. Convergence Results 2. With the notation as in Section 2, a simple difference approximation to (1.2) is

$$
\begin{gathered}
u_{n+1}-2 u_{n}+u_{n-1}=h^{2} f\left(t_{n}, u_{n}\right), \quad n=1, \cdots, N-1, \\
u_{0}=a, \quad u_{N}=b .
\end{gathered}
$$

The following theorem gives the convergence properties of a solution of (3.1) to a solution of (1.2), as $h \rightarrow 0$. By a solution of the differential equation, we mean a function $y(t)$ which has an absolutely continuous first derivative on $[0,1]$ satisfies the boundary condition, and $y^{\prime \prime}(t)$ equals $f$, except on a set of Lebesgue measure zero. The proof of the theorem is similar to that of Theorem 2.1; we only sketch the differences.

THEOREM 3.1. Suppose the solution to (1.2) exists, where $f$ is a bounded Riemann integrable function along the solution trajectory. Assume, in addition, there exists $K<8$ such that for all $t \in[0,1]$ and $x, y$ real

$$
|f(t, x)-f(t, y)| \leqq K|x-y| .
$$

Then

$$
\left|u_{n}-y\left(t_{n}\right)\right| \rightarrow 0 \text { uniformly, as } h \rightarrow 0, n h \rightarrow t \in[0,1] \text {, }
$$

where $y(t)$ is the exact solution of the differential equation and $u_{n}$ is the solution to (3.1). If, in addition, $f$ is of bounded variation along the solution trajectory, then

$$
\left|u_{n}-y\left(t_{n}\right)\right|=O(h) .
$$

Proof. Define the local truncation error $\tau_{n}$ by

$$
h^{2} \tau_{n}=y\left(t_{n+1}\right)-2 y\left(t_{n}\right)+y\left(t_{n-1}\right)-h^{2} f\left(t_{n}, y\left(t_{n}\right)\right),
$$

$n=1, \cdots, N-1$. Set $v$ to be the vector with components $v_{n}=y\left(t_{n}\right)-u_{n}, n=1$, $\cdots, N-1$. Then, from (3.5), $v$ satisfies

$$
v=-h^{2} A^{-1} F-h^{2} A^{-1} \tau,
$$

where $A^{-1}=\left(r_{i j}\right), i, j=1, \cdots, N-1$, has elements

$$
\begin{aligned}
r_{i i}= & \frac{i(N-j)}{N}, \quad i \leqq j, \\
& =r_{i i}, \quad i>j,
\end{aligned}
$$

$F$ has components $F_{n}=f\left(t_{n}, y\left(t_{n}\right)\right)-f\left(t_{n}, u_{n}\right), n=1, \cdots, N-1$, and $\tau$ has components $\tau_{n}, n=1, \cdots, N-1$. From (3.6), it follows, by using (3.2), that

$$
\|v\|_{\infty} \leqq \frac{2}{8-K} \sum_{i=1}^{N-1} h\left|\tau_{i}\right| .
$$

Integration by parts shows that 


$$
\frac{y\left(t_{n+1}\right)-2 y\left(t_{n}\right)+y\left(t_{n-1}\right)}{h}=\int_{0}^{h}\left[y^{\prime \prime}\left(t_{n}+\theta\right)+y^{\prime \prime}\left(t_{n}-\theta\right)\right]\left(1-\frac{\theta}{h}\right) d \theta .
$$

Then using this, (3.5) and (1.2), we obtain

$$
\sum_{n=1}^{N-1} h\left|\tau_{n}\right| \leqq \frac{h}{2} \sum_{n=1}^{N-1}\left(M_{n}-m_{n}\right)+\frac{h}{2} \sum_{n=1}^{N-1}\left(M_{n-1}-m_{n-1}\right),
$$

where

$$
m_{n}=\inf _{t \in[0, h]} f\left(t_{n}+t, y\left(t_{n}+t\right)\right), \quad n=0,1, \cdots, N-1,
$$

and

$$
M_{n}=\sup _{t \in[0, h]} f\left(t_{n}+t, y\left(t_{n}+t\right)\right), \quad n=0,1, \cdots, N-1 .
$$

Then, as in Theorem 2.1, the conclusions follow.

Department of Applied Mathematics and Computer Science

University of Virginia

Charlottesville, Virginia 22901

1. E. A. Coddington \& N. Levinson, Theory of Ordinary Differential Equations, McGraw-Hill, New York, 1955. MR 16, 1022.

2. G. G. DAHLQUIST, Stability and Error Bounds in the Numerical Integration of Ordinary Differential Equations, Kungl. Tekn. Högsk. Stockholm, No. 130, 1959. MR 21 \#1706.

3. R. Goodman, Round-Off Error in the Numerical Solution of Retarded Ordinary Differential Equations, Thesis, Harvard University, Cambridge, Mass., 1971.

4. P. HeNRICI, Discrete Variable Methods in Ordinary Differential Equations, Wiley, New York, 1962. MR 24 \#B1772.

5. P. HeNRICI, Error Propagation for Difference Methods, Wiley, New York, 1963. MR 27 \#4365.

6. M. LeEs, Discrete Methods for Nonlinear Two-Point Boundary Value Problems, Proc. Sympos. Numerical Solution of Partial Differential Equations (Univ. of Maryland, 1965), Academic Press, New York, 1966, pp. 59-72. MR 34 \#2196.

7. T. S. ZVERKINA, "Approximate solution of differential equations with retarded argument and differential equations with discontinuous right-hand sides," Trudy Sem. Teor. Differencial. Uravneniǐ s Otklon. Argumentom Univ. Družby Narodov Patrisa Lumumby, v. 1, 1962, pp. 76-93. (Russian) MR 32 \#2708. 